\title{
Erratum
}

\section{Erratum: Nomi et al., "Moment-to-Moment BOLD Signal Variability Reflects Regional Changes in Neural Flexibility across the Lifespan"}

In the article "Moment-to-Moment BOLD Signal Variability Reflects Regional Changes in Neural Flexibility across the Lifespan" by Jason S. Nomi, Taylor S. Bolt, C.E. Chiemeka Ezie, Lucina Q. Uddin, and Aaron S. Heller, which appeared on pages 5539-5548 of the May 31, 2017 issue, there were two errors related to data analysis. The authors state the following:

There was an error in the MATLAB code used to calculate mean square successive differences (MSSD). The square root of MSSD ${ }^{2}$ was not computed in the original set of analyses. Thus, the paper reports analyses using the measure MSSD ${ }^{2}$ (MSSD squared) instead of MSSD. Second, the command "fslcc" was used to correlate within-subject whole-brain variability maps between MSSD and SD. However, fslcc includes empty voxels with zeros for calculating the correlation between two 3-D nifti files, which artificially inflates effect sizes. All data have been reanalyzed, and the main conclusions of the paper are unaffected.

With regard to reporting MSSD $^{2}$ instead of MSSD, because this is only an issue of variable scaling, the rank-order correlations of $\mathrm{MSSD}^{2}$ responsible for the results presented in the original manuscript remain the same for MSSD. Reanalyzing all data using MSSD instead of $\mathrm{MSSD}^{2}$ produces all of the same results and conclusions from the original paper. The sole effect that does not surpass the original significance thresholds is the positive quadratic result observed in the thalamus (Fig. 5, top) for the fast TR group ( $p=0.065$ instead of $p<0.05$ voxelwise, $p<0.05$ cluster-wise); the positive quadratic thalamus result is still significant for the slow TR group ( $p<$ 0.05 voxelwise, $p<0.05$ cluster-wise). Additionally, the post hoc regression model for the linear MSSD decrease for the sensorimotor overlap effect in the slow TR group (Table 1) that previously was not significant $(p=0.0776)$, is now significant $(p=0.000092)$.

With regards to using the fslcc command, reanalysis using custom MATLAB scripts showed that our measure of MSSD calculated from a $z$-normalized time series is negatively correlated with SD calculated from the same non-normalized time series in both the fast and slow TR groups ( $r$ values $<-0.39$; see below). This is in contrast to previous research showing that MSSD and SD are highly positively correlated ( $r$ values $>0.97$; Garret et al., 2011). However, that study calculated MSSD and SD from a percentage-change normalized time series with a mean of 100 . After calculating MSSD and SD using a percentage-change normalized time series with a mean of 100 , as done previously, we also find that percentage-change normalized MSSD and percentage-change normalized SD are highly positively correlated in both the slow and fast TR groups in our data ( $r$ values $>0.98$; see below). Future research will need to explore differences in $z$-scored normalized MSSD and percentage-change normalized MSSD.

These minor differences do not change the primary conclusions and results reported in the paper showing that in general, most brain areas exhibit decreases in MSSD across the lifespan, with the exception of increases in MSSD across the lifespan for the right dorsal anterior insula and the ventral temporal cortex. All analyses from the original paper have been replicated below and all directional relationships are unaffected.

As a result of these errors, the following changes have been made to the online version:

Update to the first paragraph of the Results section on page 5542: The average whole-brain MSSD value across all subjects was 0.2073 $(\mathrm{SD}=0.0102$, range: $0.1734-0.2359)$ for the fast TR group and 0.443 ( $\mathrm{SD}=0.0194$, range: $0.3916-0.4886)$ for the slow TR group, indicating significantly smaller MSSD for the fast TR group $\left(t_{(376)}=148.581, p<0.0001\right)$.

New text to be inserted between the first and second paragraphs of the Results section on page 5542: Within-subject voxelwise whole-brain group average correlations between $z$-score normalized MSSD (zMSSD), non-normalized SD, percentage-change normalized MSSD with a mean of 100 (\%MSSD), and percentage-change normalized SD with a mean of 100 (\%SD) showed that SD, $\%$ MSSD, and \%SD were strongly positively correlated with each other ( $r$ values $>0.98$; Table 2$)$ and negatively correlated with $z M S S D$ $(r$ values $<-0.25)$.

Update to the first paragraph of the subsection "Linear relationship between MSSD values for subjects in bothTR groups" of the Results section on page 5544: Spearman's rank-order correlations were recalculated using MSSD values for the 177 subjects common to both groups for all post hoc analyses. Significant positive correlations were replicated for all post hoc regression effects as in the original manuscript (left VTC linear increase: $\rho_{(175)}=0.291, p=0.000085$; right dAI linear increase: $\rho_{(175)}=0.470, p=3.9022 \times 10^{-11}$; sensorimotor linear decrease: $\rho_{(175)}=0.634, p=2.7058 \times 10^{-21}$; visual linear decrease: $\rho_{(175)}=0.685, p=6.9544 \times 10^{-26}$; thalamus linear decrease: $\rho_{(175)}=0.595, p=2.5408 \times 10^{-18}$; basal ganglia linear decrease: $\rho_{(175)}=0.361, p=7.8534 \times 10^{-7}$; right VTC negative quadratic: $\left.\rho_{(175)}=0.425, p=3.7767 \times 10^{-9}\right)$.

Figures 2-5 have been corrected to reproduce all of the directional results found for each brain area from the original paper, with the exception of Figure 5. The positive quadratic effect for the thalamus found in the top half of Figure 5 from the original article has been removed.

The legend for Figure 5 has been updated to read: Negative quadratic effect overlap for both TR groups with scatterplots showing MSSD effects (voxelwise at $p<0.05$ and cluster corrected at $p<0.05$ for both TR groups). Blue circles are males and red circles are females. 
Scatterplots show MSSD values for the fast TR group effect (voxelwise at $p<0.05$ ) and slow TR group effect (voxelwise at $p<0.05$ and cluster corrected at $p<0.05)$.

Table 1 has been corrected online and Table 2 has been added.

\section{Reference}

Garrett DD, Kovacevic N, McIntosh AR, Grady CL (2011) The importance of being variable. J Neurosci 31:4496-4503.

DOI: $10.1523 / J N E U R O S C I .2779-19.2019$

Table 1. Results from post hoc regression analyses

\begin{tabular}{|c|c|c|c|c|c|c|c|c|c|c|}
\hline & \multicolumn{5}{|c|}{ Fast TR group, $0.645 \mathrm{~s}$} & \multicolumn{5}{|c|}{ Slow TR group, $1.4 \mathrm{~s}$} \\
\hline & & $R^{2}$ & $(\mathrm{df}) F$ & $\beta_{\text {linear }}(p$ value $)$ & $\beta_{\text {quadratic }}(p$ value) & & $R^{2}$ & $(\mathrm{df}) F$ & $\beta_{\text {linear }}(p$ value $)$ & $\beta_{\text {quadratic }}(p$ value) \\
\hline \multirow[t]{2}{*}{ Left VTC linear increase } & M1 & 0.189 & $(6,184) 7.15$ & $\begin{array}{l}0.389 \\
\left(4.3463 \times 10^{-7}\right)\end{array}$ & - & M1 & 0.163 & $(6,180) 5.83$ & $\begin{array}{l}0.323 \\
(0.000061)\end{array}$ & - \\
\hline & M2 & 0.204 & $(7,183) 6.71$ & $\begin{array}{l}0.410 \\
\left(1.2892 \times 10^{-7}\right)\end{array}$ & $\begin{array}{l}-0.140 \\
(0.063)\end{array}$ & M2 & 0.170 & $(7,179) 5.24$ & $\begin{array}{l}0.335 \\
(0.000037)\end{array}$ & $\begin{array}{l}-0.091 \\
(0.214)\end{array}$ \\
\hline Right insula linear increase & M1 & 0.143 & $(6,184) 5.13$ & $\begin{array}{l}0.308 \\
(0.00013)\end{array}$ & - & M1 & 0.114 & $(6,180) 3.86$ & $\begin{array}{l}0.3 \\
(0.00039)\end{array}$ & - \\
\hline \multirow[t]{2}{*}{ Sensorimotor linear decrease } & M1 & 0.168 & $(6,184) 6.17$ & $\begin{array}{l}-0.352 \\
(0.002)\end{array}$ & - & M1 & 0.120 & $(6,180) 4.09$ & $\begin{array}{l}-0.356 \\
(0.000092)\end{array}$ & - \\
\hline & M2 & 0.180 & $(7,183) 5.72$ & $\begin{array}{l}-0.382 \\
(0.001)\end{array}$ & $\begin{array}{l}0.125 \\
(0.102)\end{array}$ & M2 & 0.121 & $(7,179) 3.52$ & $\begin{array}{l}-0.358 \\
(0.000088)\end{array}$ & $\begin{array}{l}0.033 \\
(0.660)\end{array}$ \\
\hline Visual linear decrease & M1 & 0.222 & $(6,184) 8.78$ & $\begin{array}{l}-0.429 \\
\left(1.9072 \times 10^{-7}\right)\end{array}$ & - & M1 & 0.173 & $(6,180) 6.28$ & $\begin{array}{l}-0.360 \\
(0.000009)\end{array}$ & - \\
\hline Thalamus linear decrease & M2 & 0.183 & $(7,183) 5.84$ & $\begin{array}{l}-0.431 \\
\left(2.1183 \times 10^{-7}\right)\end{array}$ & $\begin{array}{l}0.139 \\
(0.065)\end{array}$ & M2 & 0.138 & $(7,179) 4.08$ & $\begin{array}{l}-0.257 \\
(0.005)\end{array}$ & $\begin{array}{l}0.132 \\
(0.083)\end{array}$ \\
\hline \multirow[t]{2}{*}{ Basla ganglia linear decrease } & M1 & 0.116 & $(6,184) 4.03$ & $\begin{array}{l}-0.376 \\
(0.000006)\end{array}$ & $\overline{0.116}$ & M1 & 0.113 & $(6,180) 3.83$ & $-0.368(0.00001)$ & - \\
\hline & M2 & 0.127 & $(7,183) 3.79$ & $\begin{array}{l}-0.387 \\
(0.000004)\end{array}$ & $(0.136)$ & M2 & 0.115 & $(7,179) 3.33$ & $\begin{array}{l}-0.373 \\
(0.000008)\end{array}$ & $\begin{array}{l}0.048 \\
(0.528)\end{array}$ \\
\hline Right VTC negative quadratic & M3 & 0.197 & $(7,183) 6.40$ & $\begin{array}{l}-0.203 \\
(0.015)\end{array}$ & $\begin{array}{l}-0.233 \\
(0.003)\end{array}$ & M3 & 0.093 & $(7,179) 2.63$ & $\begin{array}{l}-0.078 \\
(0.354)\end{array}$ & $\begin{array}{l}-0.270 \\
(0.001)\end{array}$ \\
\hline
\end{tabular}

Table 2. Within-subject voxelwise whole-brain group average correlations between zMSSD, non-normalized SD, percentage-change normalized MSSD with a mean of 100 (\%MSSD), and percentage-change normalized SD with a mean of 100 (\%SD)

\begin{tabular}{|c|c|c|c|c|c|c|c|c|}
\hline & \multicolumn{4}{|c|}{ Fast TR group } & \multicolumn{4}{|c|}{ Slow TR group } \\
\hline & ZMSSD & SD & $\% M S S D$ & $\% S D$ & ZMSSD & SD & $\% M S S D$ & $\% S D$ \\
\hline ZMSSD & - & - & - & - & - & - & - & - \\
\hline SD & -0.392 & & & & -0.44 & & & \\
\hline$\% M S S D$ & -0.251 & 0.9811 & & & -0.314 & 0.9838 & & \\
\hline$\% S D$ & -0.392 & 1 & 0.9811 & & -0.439 & 0.9999 & 0.9839 & \\
\hline
\end{tabular}

SD, \%SD, and \%MSSD are strongly correlated with each other and negatively correlated with zMSSD. 


\section{Linear Increase}
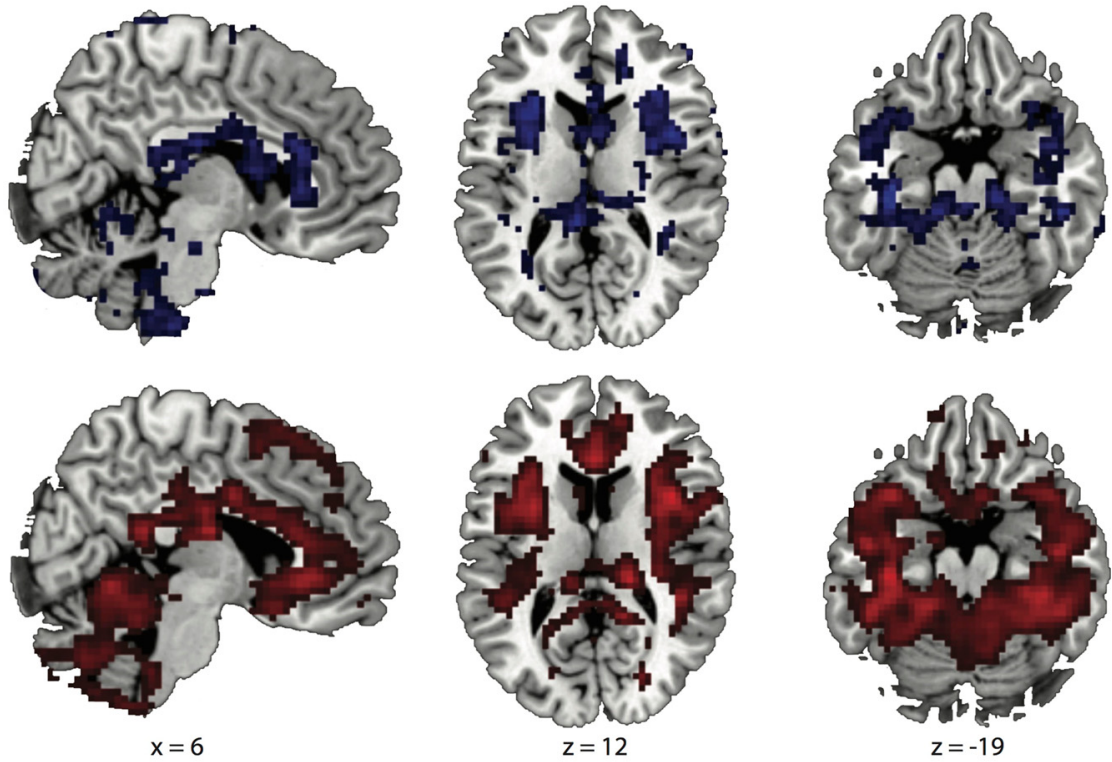

\section{Linear Decrease}
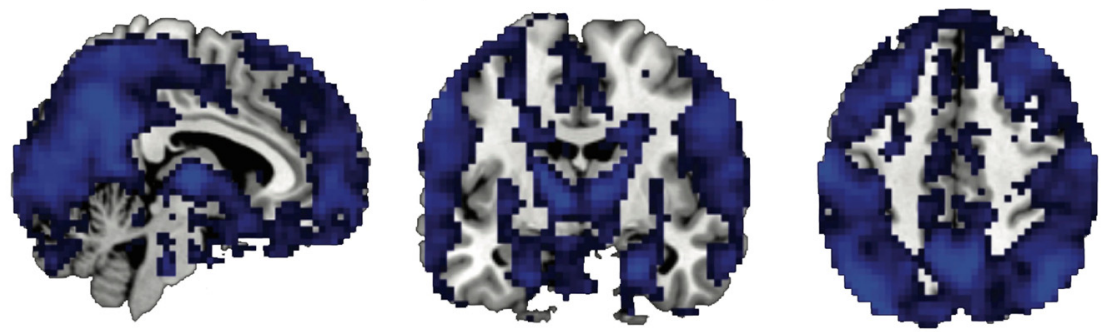

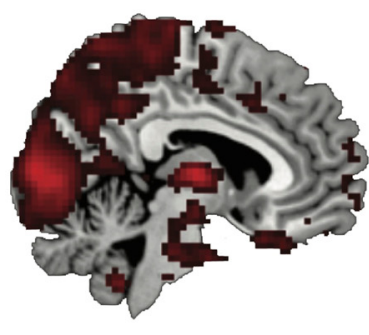

$x=6$

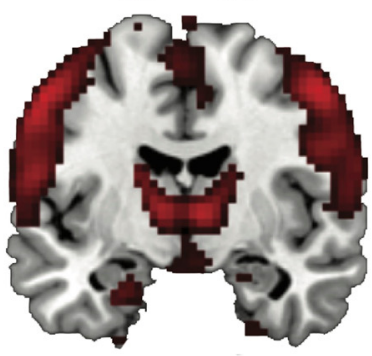

$y=-8$

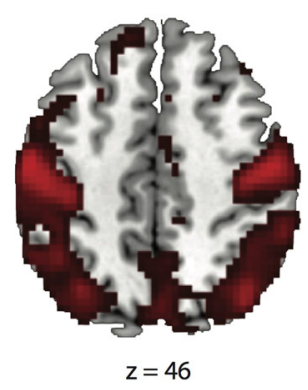

\begin{tabular}{ccc} 
& Fast TR Group $(0.645 \mathrm{sec})$ \\
\hline 0 & $\mathrm{t}$ & 5
\end{tabular}

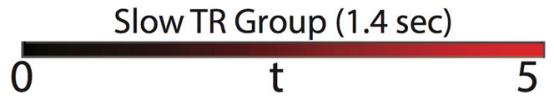

Figure 2 


\section{Linear Increase}
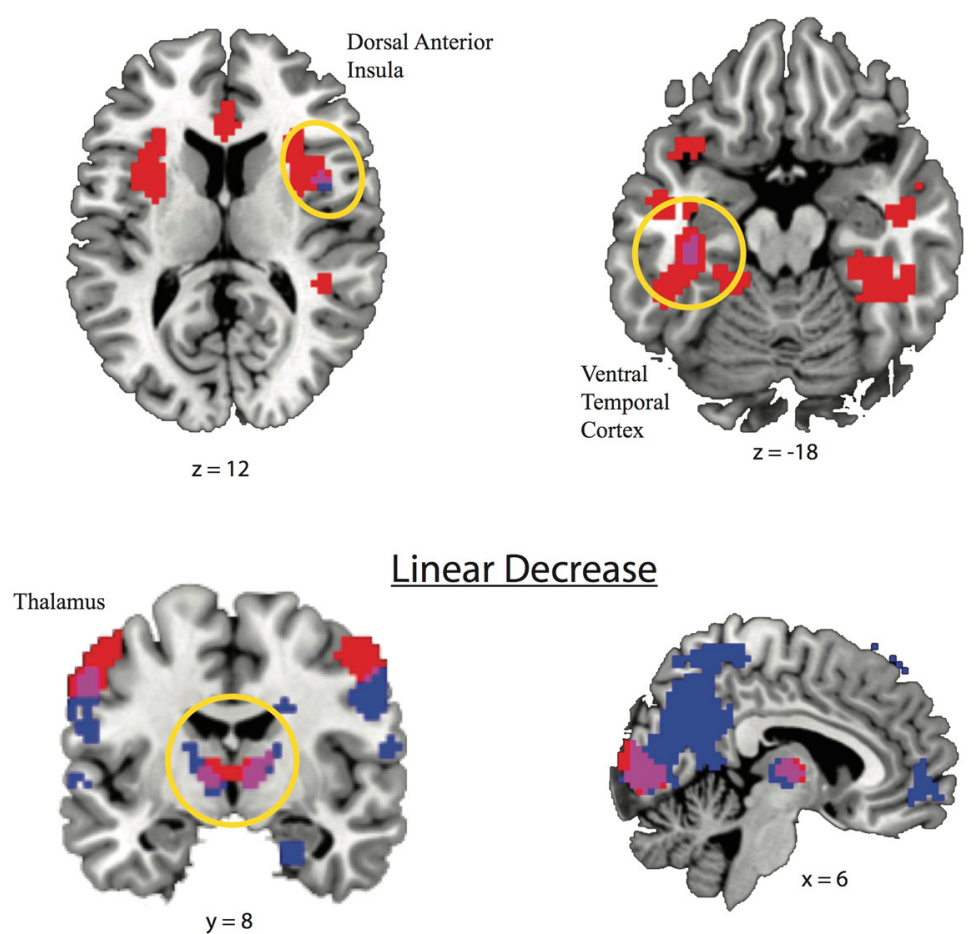

\section{Linear Decrease}
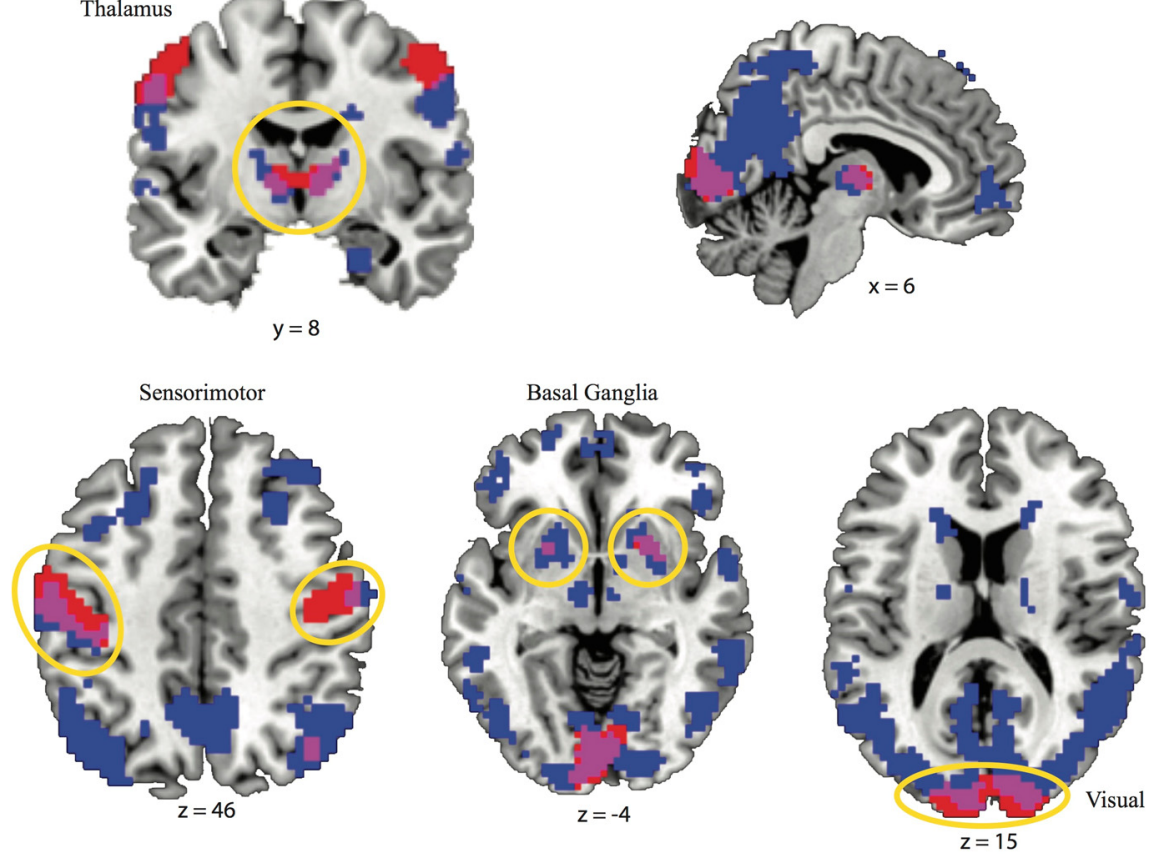

Figure 3 

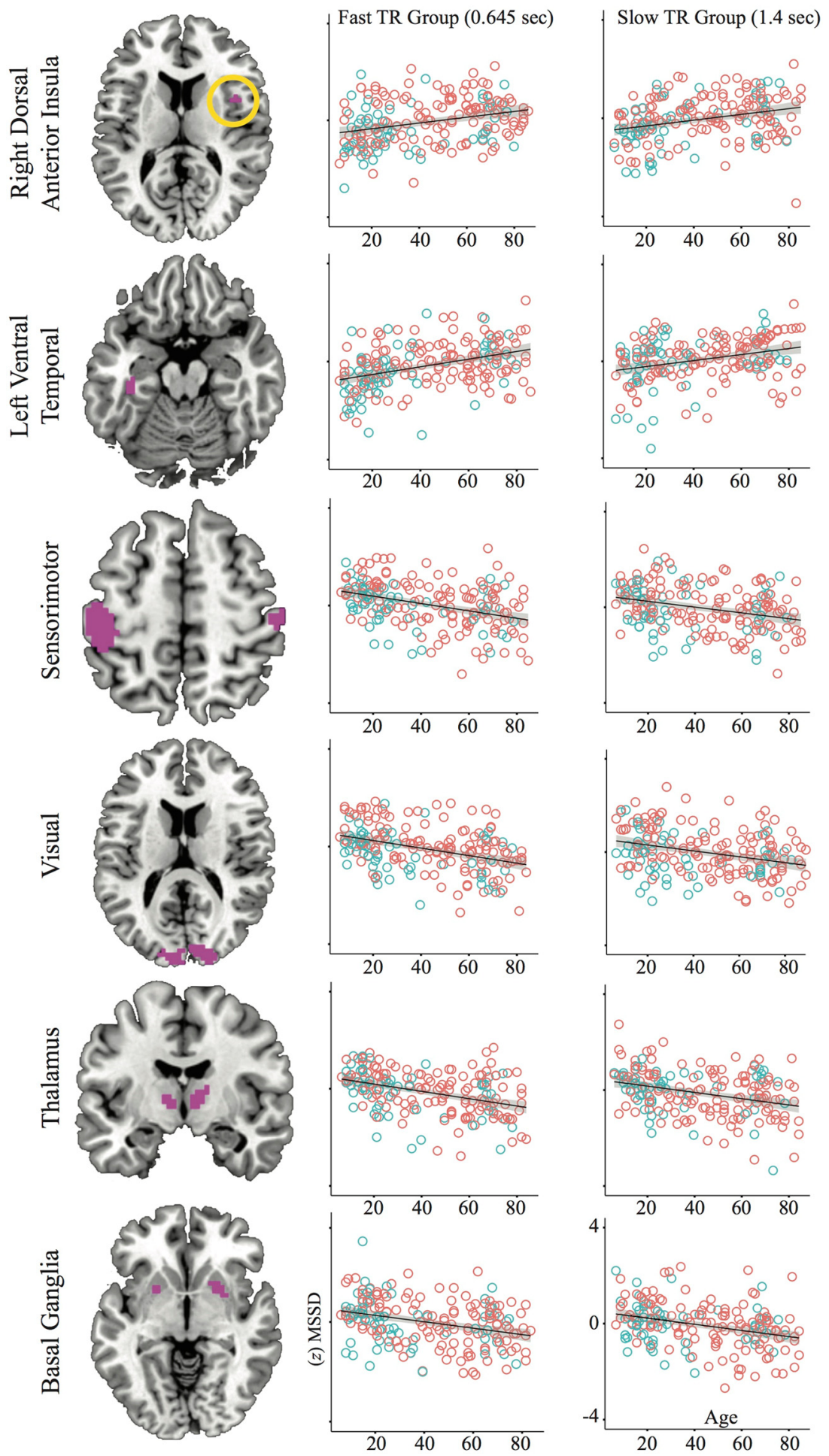

Figure 4 


\section{Negative Quadratic Group Overlap: \\ Ventral Temporal Cortex}

$\square=$ Negative quadratic slow TR group

= = Group overlap
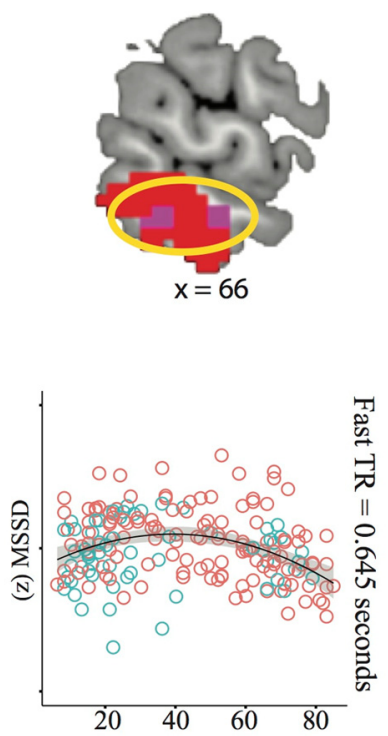

= = Negative quadratic fast TR group
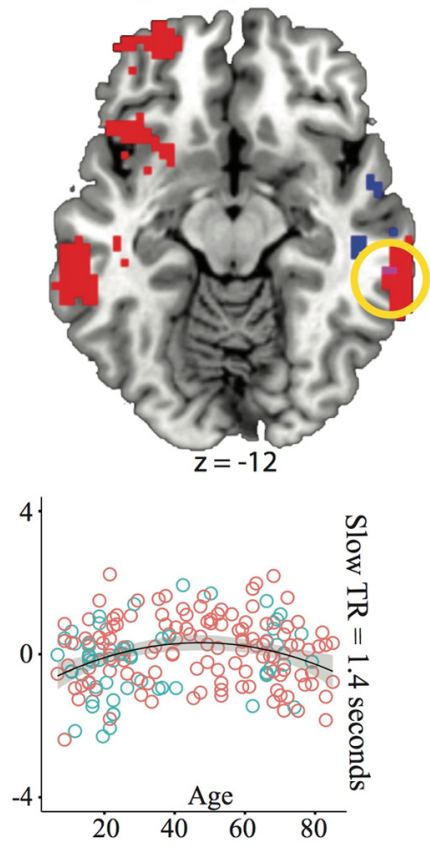

Figure 5. Negative quadratic effect overlap for both TR groups with scatterplots showing MSSD effects (voxelwise at $p<0.05$ and cluster corrected at $p<0.05$ for both TR groups). Blue circles are males and red circles are females. Scatterplots show MSSD values for the fast TR group effect (voxelwise at $p<0.05$ ) and slow TR group effect (voxelwise at $p<0.05$ and cluster corrected at $p<$ $0.05)$. 